\title{
Validation of Backscatter Measurements from the Advanced Scatterometer on $\mathrm{MetOp}-\mathrm{A}$
}

\author{
C. Anderson, J. Figa, H. BonekAmp, And J. J. W. Wilson \\ EUMETSAT, Darmstadt, Germany \\ J. Verspeek AND A. STOFFELEN \\ KNMI, De Bilt, Netherlands \\ M. Portabella \\ Unitat de Tecnologia Marina (UTM-CSIC), Barcelona, Spain
}

(Manuscript received 9 February 2011, in final form 25 July 2011)

\begin{abstract}
The Advanced Scatterometer (ASCAT) on the Meteorological Operational (MetOp) series of satellites is designed to provide data for the retrieval of ocean wind fields. Three transponders were used to give an absolute calibration and the worst-case calibration error is estimated to be $0.15-0.25 \mathrm{~dB}$.

In this paper the calibrated data are validated by comparing the backscatter from a range of naturally distributed targets against models developed from European Remote Sensing Satellite (ERS) scatterometer data.

For the Amazon rainforest it is found that the isotropic backscatter decreases from -6.2 to $-6.8 \mathrm{~dB}$ over the incidence angle range. The ERS value is around $-6.5 \mathrm{~dB}$. All ASCAT beams are within $0.1 \mathrm{~dB}$ of each other. Rainforest backscatter over a 3-yr period is found to be very stable with annual changes of approximately $0.02 \mathrm{~dB}$.

ASCAT ocean backscatter is compared against values from the C-band geophysical model function (CMOD-5) using ECMWF wind fields. A difference of approximately $0.2 \mathrm{~dB}$ below $55^{\circ}$ incidence is found. Differences of over $1 \mathrm{~dB}$ above $55^{\circ}$ are likely due to inaccuracies in CMOD-5, which has not been fully validated at large incidence angles. All beams are within $0.1 \mathrm{~dB}$ of each other.

Backscatter from regions of stable Antarctic sea ice is found to be consistent with model backscatter except at large incidence angles where the model has not been validated. The noise in the ice backscatter indicates that the normalized standard deviation of the backscatter values $K_{p}$ is around $4.5 \%$, which is consistent with the expected value.

These results agree well with the expected calibration accuracy and give confidence that the calibration has been successful and that ASCAT products are of high quality.
\end{abstract}

\section{Introduction}

The Advanced Scatterometer (ASCAT) is a European spaceborne C-band radar instrument carried on the Meteorological Operational (MetOp)-A satellite, which was launched in October 2006 (Figa-Saldaña et al. 2002; Klaes et al. 2007). The instrument is designed to accurately measure the radar backscatter from the surface of the earth. Over the ocean surface the backscatter characteristics are primarily influenced by the

Corresponding author address: Craig Anderson, EUMETSATAllee 1, EUMETSAT, D-64295 Darmstadt, Germany.

E-mail: craig.anderson@eumetsat.int wind speed and direction and, hence, ocean wind vector information can be inferred from the radar measurements.

The main purpose of ASCAT is to provide estimates of the ocean wind vector to be exploited in weather forecasting and nowcasting, ocean modeling, and climate research applications. Operational wind services have been set up in the framework of the European Organisation for the Exploitation of Meteorological Satellites (EUMETSAT) Polar System application ground segment. The ASCAT instrument is also exploited in other operational applications, such as soil moisture retrieval (Bartalis et al. 2007) and sea ice mapping and drift measurements (Lavergne et al. 2010). 
The accuracy of the retrieved geophysical information depends on the accuracy of the underlying radar backscatter measurements. These are expressed in terms of the normalized radar cross section (NRCS), which is the ratio of the received backscattered energy to that of an isotropic surface scatterer as given by the two-way radar equation. NRCS measurements, denoted by $\sigma_{0}$, typically vary between -35 and $-3 \mathrm{~dB}$ over the ocean for a wind speed range of $2-25 \mathrm{~m} \mathrm{~s}^{-1}$.

The complete ASCAT commissioning process is described in the ASCAT Calibration and Validation Plan (EUMETSAT 2004) and involves

- the setting of basic instrument and processing parameters,

- analysis of the gain patterns and calculation of calibration factors using transponders,

- validation of the backscatter from a variety of natural targets, and

- validation of retrieved ocean winds against numerical weather prediction (NWP) results and ocean buoy measurements.

The gain pattern analysis and results of the calibration are described by Wilson et al. (2010), and the validation of the retrieved ocean winds is given by Verspeek et al. (2010). Although the calibration and validation plan did not give any emphasis to cross calibrations with other scatterometers [such as European Remote Sensing Satellite $(E R S) 1 / 2$ and Quick Scatterometer (QuikSCAT)], first comparisons with ERS-2 are given by Bartalis (2009).

The aim of this paper is to provide an overview of the calibration of the ASCAT and to assess the accuracy and stability of the NRCS measurements by means of geophysical validations.

In section 2, the ASCAT instrument and ground processing is briefly described. In section 3 the external calibration with transponders is summarized and the key results on the accuracy of the $\sigma_{0}$ measurements, as elaborated by Wilson et al. (2010), are presented. Section 4 discusses the geophysical validation activities over the rainforest, open ocean, and sea ice. The latter are based on comparisons with established geophysical models. The performance of the ASCAT calibration against expectations is discussed in section 5 .

\section{ASCAT instrument and processing}

The ASCAT instrument, described by Gelsthorpe et al. (2000), is the follow-on scatterometer for the Active Microwave Instruments (AMI) on ERS-1 and -2. Like these, ASCAT operates at a frequency in $\mathrm{C}$ band $(5.3 \mathrm{GHz})$ and the radar signal polarization is vertical $(\mathrm{VV})$. A major difference in design is that ASCAT comprises two sets of three fan-beam antennas. One set points to the left of the subsatellite track and the other to the right so that measurements from two $550-\mathrm{km}$-wide swaths located approximately $360 \mathrm{~km}$ to the left and right of the satellite ground track, and covering an incidence angle range of $25^{\circ}-65^{\circ}$, are obtained. This differs from the AMI, which has only a single set of fan-antennas covering a single swath with an incidence angle range of $19^{\circ}-55^{\circ}$

To achieve a high range resolution, ASCAT transmits long pulses (of approximately $10 \mathrm{~ms}$ ) with a linear frequency modulation at a carrier frequency of $5.225 \mathrm{GHz}$, with a peak power of about $120 \mathrm{~W}$. The received echoes are low-pass filtered, demodulated, and Fourier transformed on board. The resulting spectra give the received power as a function of slant range.

Echo measurements are averaged along track on board and are passed, together with measurements of noise and internal calibration data, to the ground for further processing. The measurement mode processing consists of corrections to the raw power echoes (to remove the range-dependent receiver filter response, noise, and instrument power gain variations), normalization into NRCS values, and finally spatial averaging to obtain triplets of $\sigma_{0}$ estimates (corresponding to the three antenna beams) at the required locations. The following two products containing spatially averaged backscatter values are produced:

- Sigma Zero Operational (SZO) in which the backscatter resolution is around $50 \mathrm{~km}$ and the backscatter values are calculated at 21 locations (termed nodes or wind vector cells) across the swath (The spacing between nodes and between successive rows of nodes is approximately $25 \mathrm{~km}$.), and

- Sigma Zero Research (SZR) with a resolution of around $28 \mathrm{~km}, 41$ nodes across the swath, and a node spacing of approximately $12.5 \mathrm{~km}$.

Details of the processing and products are described in the ASCAT product generation function specification (EUMETSAT 2005) and the ASCAT product guide (EUMETSAT 2009a).

\section{External calibration}

ASCAT is calibrated by means of three transponders that have been designed to provide stable and accurately known point target cross sections. Each transponder tracks the MetOp satellite during an overpass, and when they receive the signal transmitted by the ASCAT they wait a fixed time interval before sending a signal of precisely known cross section back to it. The transponders are located in Turkey and their position was carefully 


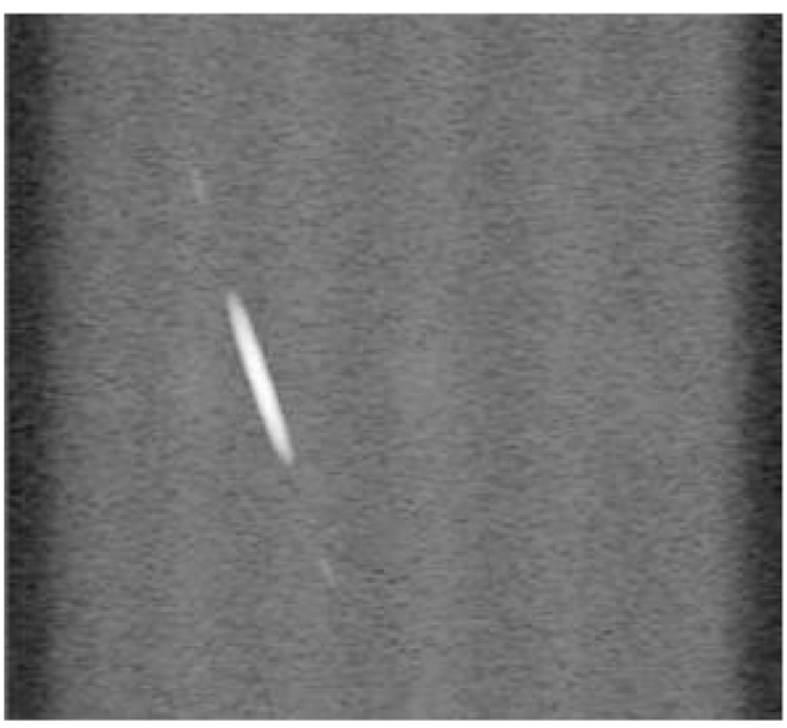

FIG. 1. Image of a typical transponder signal recorded by ASCAT.

chosen to give optimum sampling of each antenna beam during the 29-day repeat cycle of $M e t O p$ - $A$.

The calibration procedure has several steps. First, the ASCAT data containing the transponder signal is processed to give the antenna gain value in the antenna coordinate system. This gives the antenna gain on a cut through the beam pattern at a particular elevation angle. An example of the raw ASCAT data containing a transponder signal is shown in Fig. 1, and an example of the antenna gain as a function of the normalized antenna azimuth angle is shown in Fig. 2. This process is repeated for a number of passes over the transponders at various elevation angles and a well-sampled antenna gain pattern is obtained, as depicted in Fig. 3.

In the second step, a model of the antenna gain, antenna pointing error, and gain pattern distortion is fitted to the set of data points. The residual between the data and the fitted model gives an indication of calibration accuracy.

In the third step of the process, the gain pattern models are used to obtain normalization factors for converting the ASCAT measurements into absolutely calibrated backscatter. To do this we assume the earth's backscatter to be unity and use the gain patterns to estimate the signal measured by ASCAT. Any differences between the estimated and actual signal are taken to be a result of the earth's backscatter not being unity, and dividing the actual signal by the estimated signal gives an estimate of the earth's backscatter. Hence, the estimated signal is the required normalization factor. These are calculated at various locations around the $\mathrm{MetO} \mathrm{P}-\mathrm{A}$ orbit to take into account height and geometry variations.

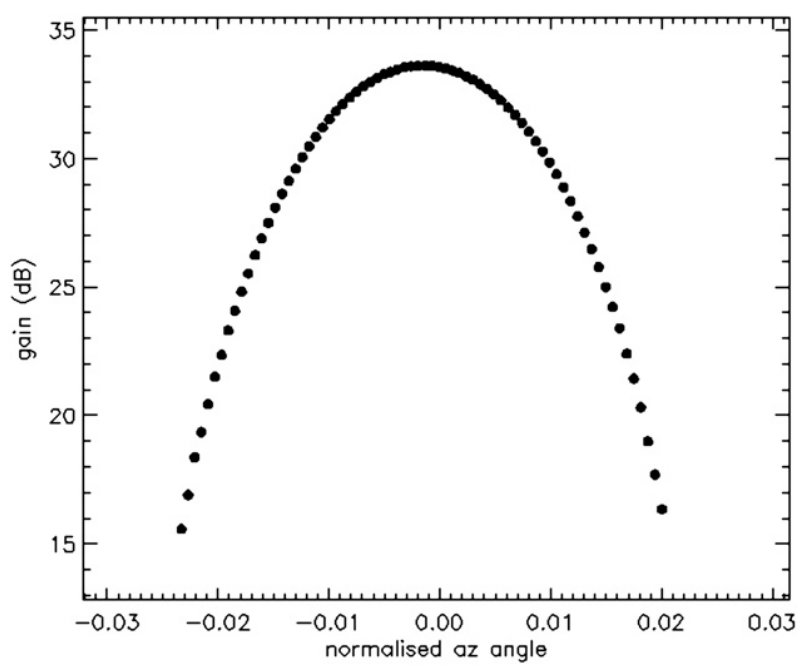

FIG. 2. Antenna gain as a function of antenna azimuth angle derived from a single pass over a transponder in the left fore beam.

Calibration campaigns, in which the transponders are operational and ASCAT is switched to calibration mode during every overpass, last approximately 2 months and are planned to take place every $18-24$ months during the ASCAT lifetime.

The first campaign took place in November and December 2006, using the single transponder that was operational at that time. This gave a preliminary calibration and allowed products to be distributed as soon after launch as possible.

The second campaign, using all three transponders, took place during winter 2007/08. The results from this campaign marked the end of the ASCAT commissioning phase and were used to reprocess older data and were also applied to the operational data. A description of this campaign and an initial investigation of the calibration quality are given in the ASCAT commissioning quality report (EUMETSAT 2009b). A more detailed report is given by Wilson et al. (2010), where an error analysis suggests a worst case around orbit calibration error of $0.15-0.25 \mathrm{~dB}$.

\section{Geophysical validations}

Geophysical validations form part of the ASCAT calibration and validation plan (EUMETSAT 2004). In these, the response from distributed natural targets is investigated to assess the quality of the backscatter. Geophysical validations can be performed over a variety of natural targets, for example, the rainforest, open ocean, sea ice, and land ice. Validations over the global ocean have been used to derive bias correction coefficients, which, when applied to the calibrated ASCAT 


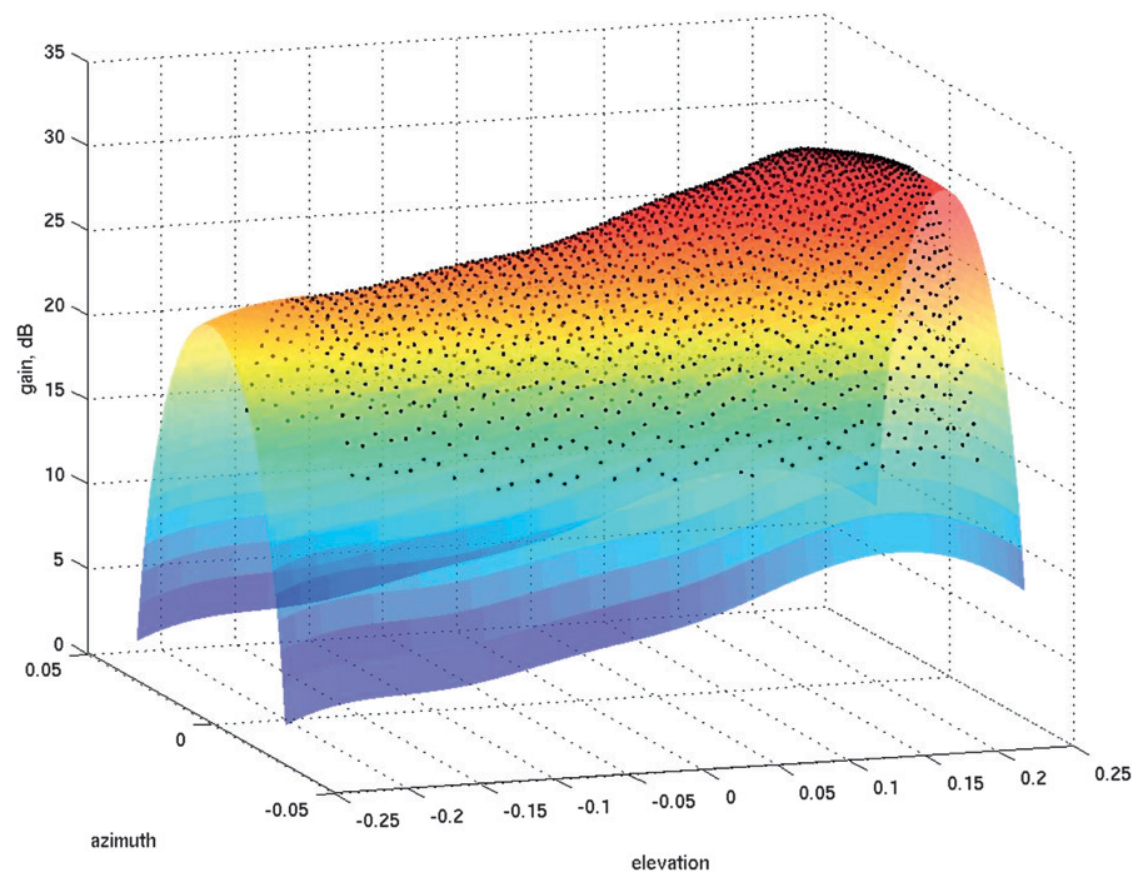

FIG. 3. Depiction of antenna gain as a function of azimuth and elevation angles produced by data from multiple passes over the transponders.

data, bring it into alignment with the ERS-based C-band geophysical model function (CMOD-5) ocean backscatter model (Verspeek et al. 2010). This was done in order to allow the retrieval of ASCAT winds soon after the MetOp launch, using only the available backscatter model. These coefficients have been also used, until recently, to generate scatterometer soil moisture values from an ERS-based model (Bartalis et al. 2007). Geophysical validations are also routinely used to monitor the quality of the backscatter data produced by the operational ASCAT processor.

In this paper we report on validation results obtained from the $50-\mathrm{km}$-resolution reprocessed backscatter data from the period $2007 / 08$ and the $50-\mathrm{km}$-resolution operational data produced during 2009. This validation dataset covers a period of $3 \mathrm{yr}$.

\section{a. Validation using rainforest backscatter}

The backscatter from areas of rainforest has been extensively studied using the ERS-1 and -2 scatterometers and has been found to be relatively stable. In particular, the isotropic backscatter given by $\gamma_{0}=\sigma_{0} / \cos \theta$ is found to be approximately constant with respect to time, viewing geometry, and spatial location. An example of this as a function of incidence angle (taken from the ERS wind scatterometer cyclic report for cycle 42 in AprilMay 1999) is shown in Fig. 4. The region of Amazon rainforest used for monitoring ERS lies within $-70^{\circ}$ and

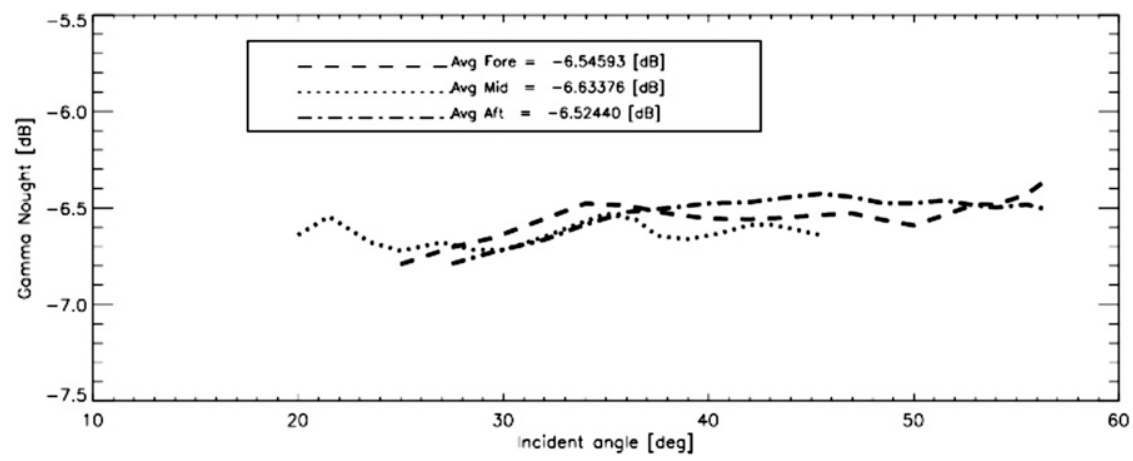

FIG. 4. An example of mean ERS $\gamma_{0}$ as a function of incidence angle. This plot is taken from the ERS wind scatterometer cyclic report for cycle 42 (April-May 1999). 


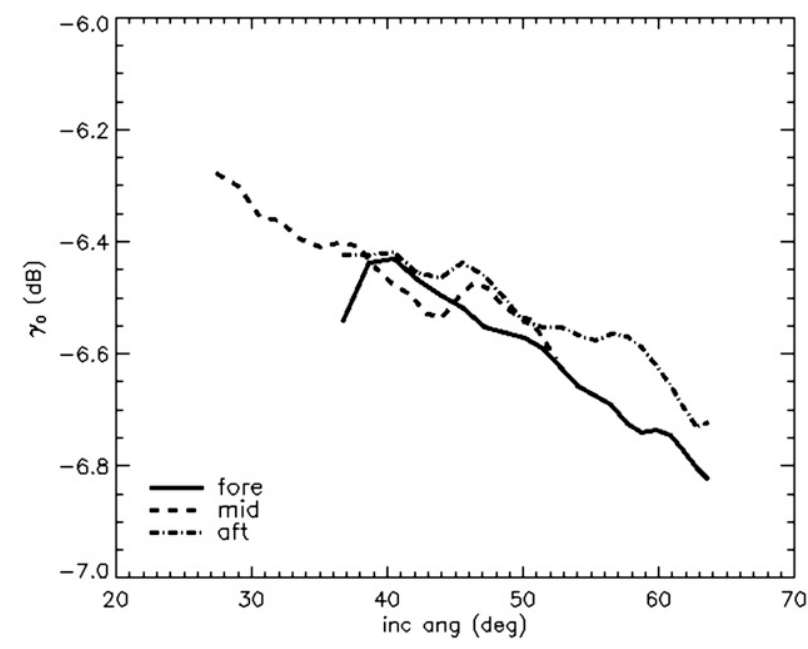

FIG. 5. Mean $\gamma_{0}$ for the left-hand beams as a function of incidence angle using descending pass data from the year 2007.

$-60.5^{\circ}$ longitude and $-2.5^{\circ}$ and $5^{\circ}$ latitude, and the value of $\gamma_{0}$ given by ERS data is approximately $-6.5 \mathrm{~dB}$. Hence, we can validate ASCAT data by taking ASCAT backscatter measurements from this region, calculating $\gamma_{0}$, and comparing it to the expected value.

Figure 5 shows the mean ASCAT $\gamma_{0}$ for the left-hand antennas as function of incidence angle using all of the descending pass data during 2007 (which gives approximately 4300 samples at each value of incidence angle). The most obvious aspect of these plots is that ASCAT $\gamma_{0}$ is not a constant value close to $-6.5 \mathrm{~dB}$, but instead decreases from approximately -6.2 to $-6.8 \mathrm{~dB}$ over the incidence angle range. The $\gamma_{0}$ values in each of the three beams are similar with differences of at most $0.1 \mathrm{~dB}$. This value does not completely represent the relative calibration between beams because it is also influenced by nonhomogeneities in the rainforest and differences in viewing geometry. The mean $\gamma_{0}$ for the right-hand antennas is shown in Fig. 6 and we find similar behavior.

These results validate ASCAT to a certain extent because the -6.2 to $-6.8 \mathrm{~dB}$ range for $\gamma_{0}$ encompasses the expected value of $-6.5 \mathrm{~dB}$. They also show that the relative calibration between beams is better than $0.1 \mathrm{~dB}$. The behavior of ASCAT $\gamma_{0}$ with incidence angle is unexpected because the $\gamma_{0}$ from the ERS data is generally considered to be approximately constant across the incidence angle range. However, other authors have found dependencies on incidence angle. For example, Zec et al. (1999) examine backscatter data from the Ku-band National Aeronautics and Space Administration (NASA) scatterometer (NSCAT) over the Amazon rainforest and model the incidence angle behavior by fitting a thirdorder polynomial. Their data show that the Ku-band backscatter over the rainforest changes from around

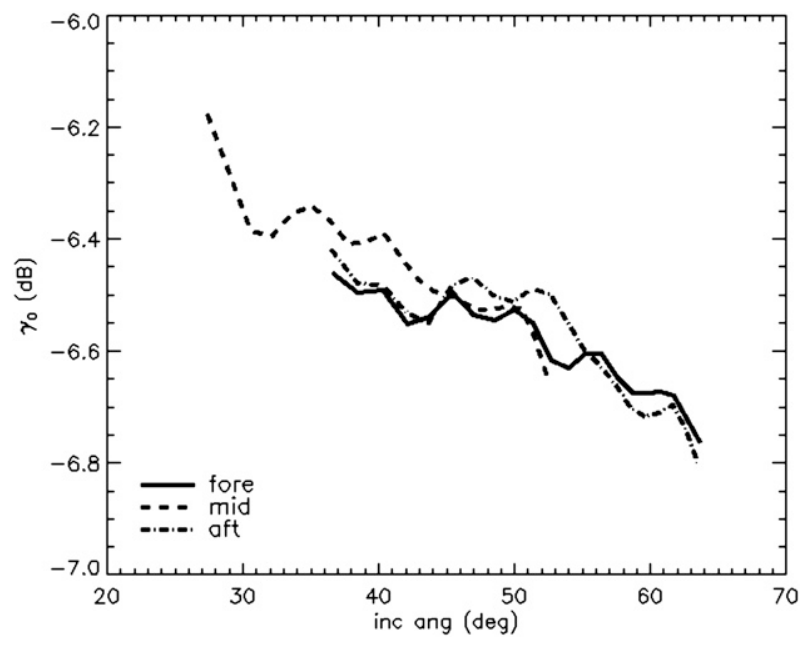

FIG. 6. As in Fig. 5, but for the right-hand beams.

-6 to $-8 \mathrm{~dB}$ over an incidence angle range of $20^{\circ}-50^{\circ}$. These values of backscatter correspond to $\gamma_{0}$ values of -5.7 and $-6.1 \mathrm{~dB}$. This gives a change in NSCAT $\gamma_{0}$ of around $-0.4 \mathrm{~dB}$ as the incidence angle increases from $20^{\circ}$ to $50^{\circ}$, and this is very similar to behavior we observe in ASCAT $\gamma_{0}$.

The stability of ASCAT is also of importance and can be examined using rainforest data. Figure 7 shows the mean $\gamma_{0}$ as a function of incidence angle for beam 1 (left midbeam) using data from the years 2007, 2008, and 2009. The difference between these is less than $0.02 \mathrm{~dB}$, which shows that both ASCAT and the annual averages of rainforest backscatter were very stable during this period.

Stability over shorter time scales is shown by the time series plot of rainforest $\gamma_{0}$ in Fig. 8. Each point in the figure shows the mean $\gamma_{0}$ at a particular incidence angle

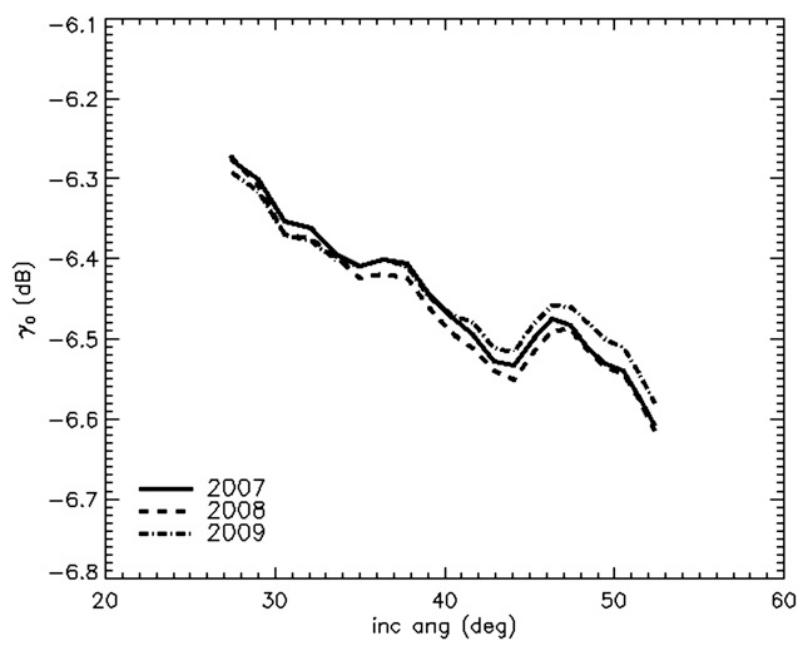

FIG. 7. Mean $\gamma_{0}$ as a function of incidence angle for the left midbeam in the years 2007, 2008, and 2009 . 


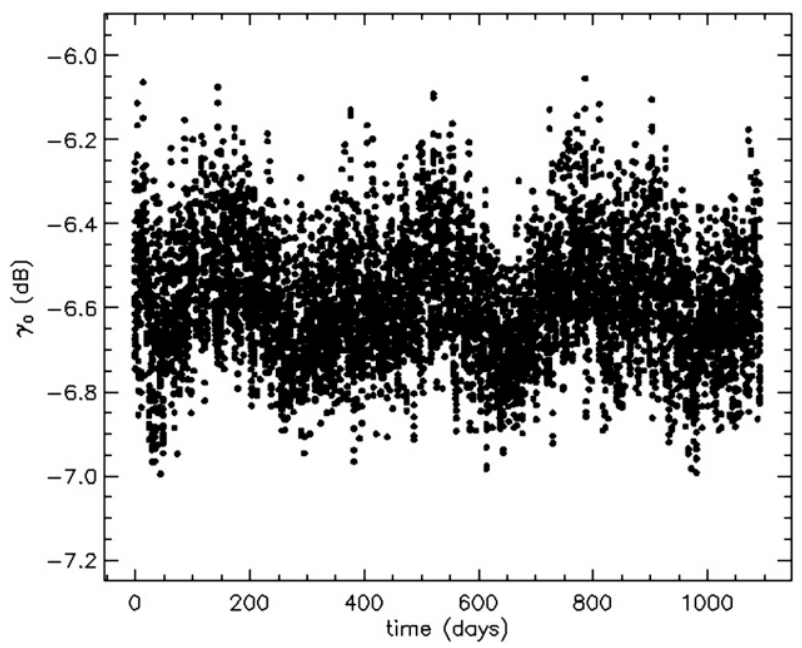

FIG. 8. Time series plot of rainforest $\gamma_{0}$ in the left midbeam for the years 2007, 2008, and 2009 .

during a pass over the rainforest. The spread in $\gamma_{0}$ values is partly due to the incidence angle effect noted earlier in which larger incidence angles have lower $\gamma_{0}$ values.

However, there is another contribution to the spread caused by inhomogeneities in the rainforest. This is demonstrated by Fig. 9, which shows the geographical location of the near-, mid-, and far-range nodes in beam 1 ascending pass data during the years 2007 and 2008 . These are not uniformly distributed across the region but cut through the rainforest at characteristic locations. Hence, different incidence angles observe different parts of the rainforest.

Figure 10 shows the mean $\gamma_{0}$ along each of the near-, mid-, and far-range lines of nodes (red, blue and green symbols) as a function of the mean longitude. The different colored symbols are displaced from each other in the vertical direction (showing variation of the $\gamma_{0}$ with incidence angle), but they also show a characteristic variation with longitude, which is caused by spatial variations in the rainforest.

Both of these factors need to be corrected in order to detect any small changes in the behavior of ASCAT. The variation with incidence angle can be reduced by adding a node-dependent bias correction so that the different colored symbols in Fig. 10 are brought into alignment. The spatial variation can be reduced by adding a longitude-dependent bias correction so that the $\gamma_{0}$ values become approximately constant. The biascorrected data (shown in Fig. 11) show very little variation with respect to either incidence angle or longitude. A time series of the bias-corrected data is shown in Fig. 12 and is less noisy than the original time series of Fig. 8. Seasonal variation in the rainforest of up to $0.2 \mathrm{~dB}$ can clearly be seen in this plot.

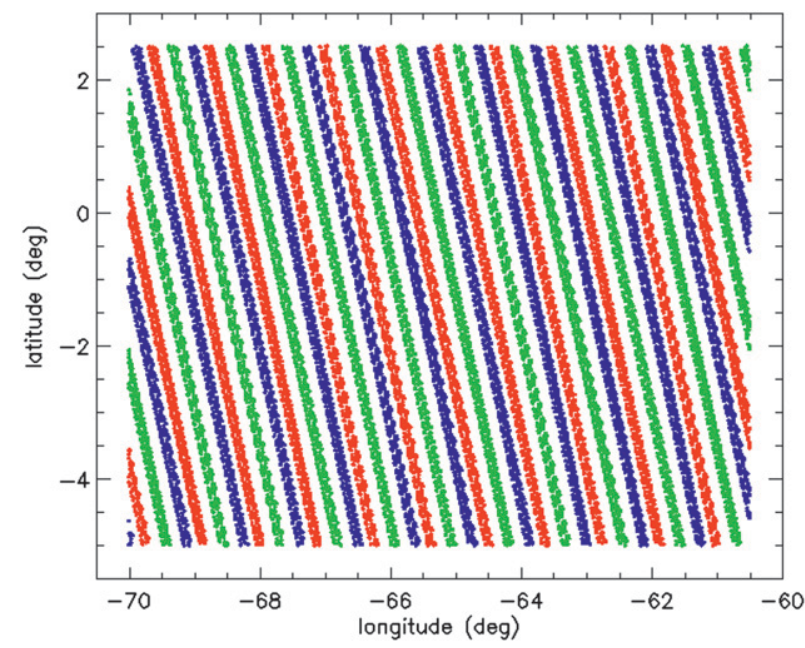

FIG. 9. The position of the near- (red), mid- (green), and farrange (blue) nodes in the left midbeam in the rainforest test site during the years 2007 and 2008 .

This method can be used to monitor the behavior of the ASCAT calibration. Figure 13 shows a time series of the rainforest $\gamma_{0}$ in the left midbeam around September 2009, and we observe an unexpected step change of approximately $0.1 \mathrm{~dB}$. This change is investigated in more detail in the next section.

The results presented in this section show that the calibrated ASCAT data over the rainforest has a similar value of $\gamma_{0}$ to ERS scatterometer data. However, the incidence angle behavior is different, pointing to some differences in the ERS and ASCAT calibrations. The reasons for this need to be understood before the merging of ERS and ASCAT data can take place to create a single dataset with consistent characteristics.

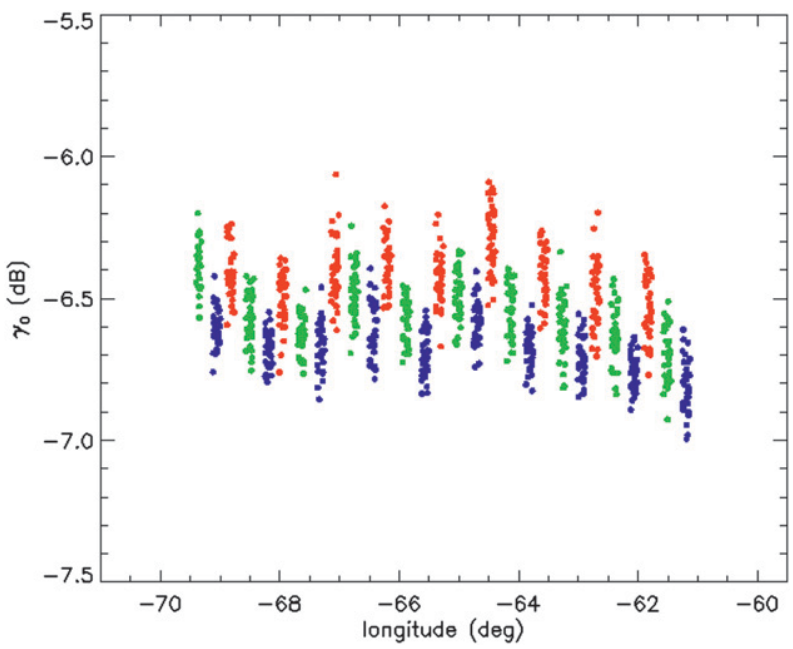

FIG. 10. Mean $\gamma_{0}$ for the near- (red), mid- (green), and far-range (blue) nodes of the left midbeam as a function of the mean longitude. 


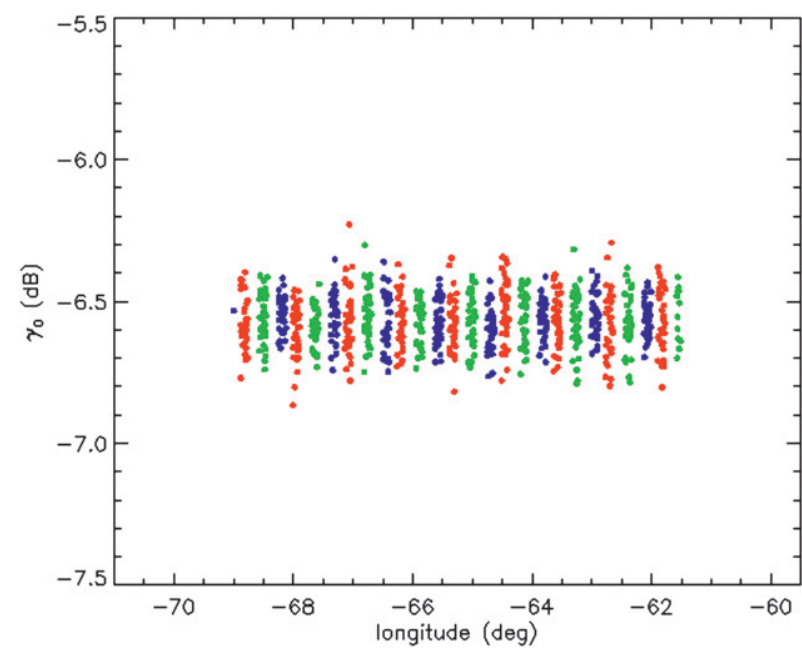

FIG. 11. As in Fig. 10, but with bias-corrected data.

The results also show that $\gamma_{0}$ values from the individual ASCAT beams are within $0.1 \mathrm{~dB}$ of each other, which is consistent with the expected calibration accuracy. Yearly averages of rainforest backscatter are also found to be very stable, with changes less than $0.02 \mathrm{~dB}$ over the period 2007-09.

\section{b. Validation using ocean backscatter}

Data from the ERS scatterometers have been used to develop a number of ocean backscatter models in which the backscatter is a function of incidence angle, wind speed, and wind direction. The latest of these are CMOD-5 (Hersbach 2003) and its equivalent neutral wind counterpart CMOD-5.n (Hersbach 2008; Verhoef et al. 2008; Portabella and Stoffelen 2009). If the wind vector over the ocean is known, either from buoy measurements or from NWP models, then the output of the ocean backscatter model can be compared to the ASCAT data. Any bias between the two indicates either a difference between the ASCAT and ERS calibrations or to different biases in the input wind vectors-CMOD-5 and operational European Centre for Medium-Range Weather Forecasts (ECMWF) input are now found to produce backscatter values that are biased low for ERS data by about $0.5 \mathrm{~dB}$ (Verhoef et al. 2008), and this may be due to a bias in the ECMWF winds, which can be roughly removed by increasing them by about $0.5 \mathrm{~m} \mathrm{~s}^{-1}$. Variations on this approach have been developed and used by the Ocean and Sea Ice Satellite Application Facility (OSI SAF), for example, the NWP ocean calibration (NOC) and visual ocean calibration (VOC) methods (Verspeek et al. 2010).

Figure 14 shows the mean difference between the backscatter produced by CMOD-5 with ECMWF winds

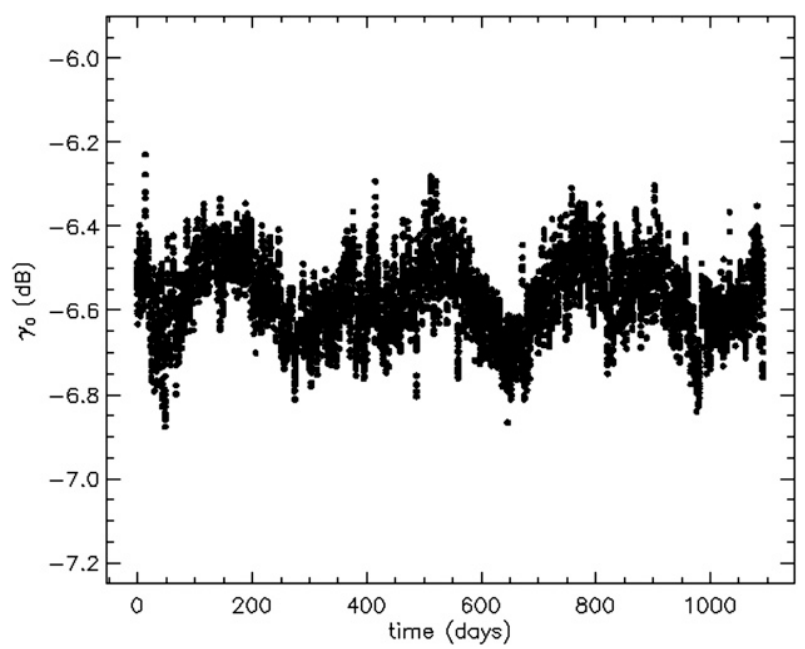

FIG. 12. Bias-corrected time series plot of rainforest $\gamma_{0}$ in the left midbeam for the years 2007-09.

and ASCAT data over the open ocean during July 2009. The plots agree strongly with the results presented by Verspeek et al. (2010) and show two distinct types of behavior.

First, between $30^{\circ}$ and $55^{\circ}$ incidence the mean difference between ASCAT- and the ERS-based CMOD-5 is approximately constant at about $0.2 \mathrm{~dB}$. This contrasts with the rainforest validation shown in the previous section, which implies that the difference between ASCAT and ERS calibrations varies with the incidence angle.

Second, above $55^{\circ}$ incidence the difference rises rapidly to about $1 \mathrm{~dB}$. However, because CMOD-5 was developed from ERS data covering the incidence angle range $19^{\circ}-55^{\circ}$, it seems likely that this is a result of inaccuracies in CMOD-5 when extrapolated to large

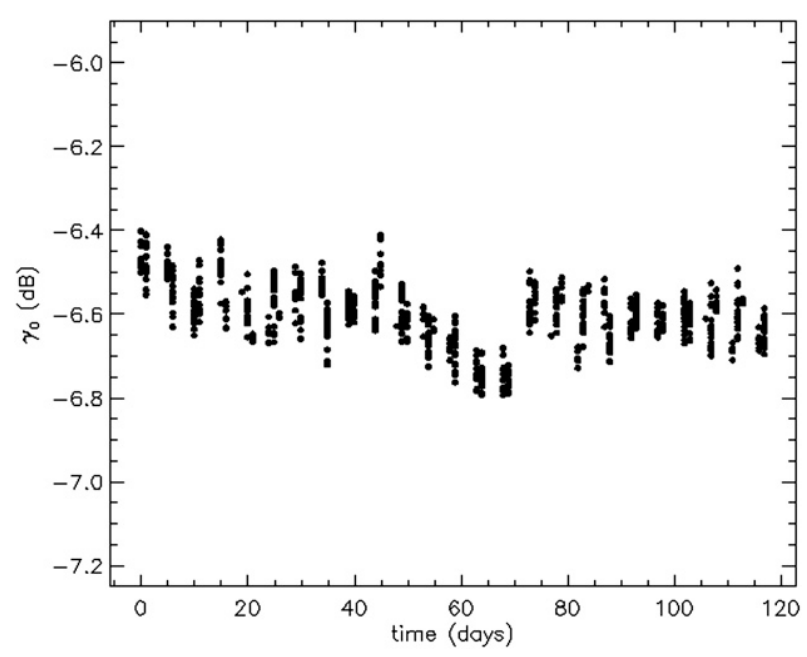

FIG. 13. Bias-corrected time series of $\gamma_{0}$ in the left midbeam for July-October 2009 


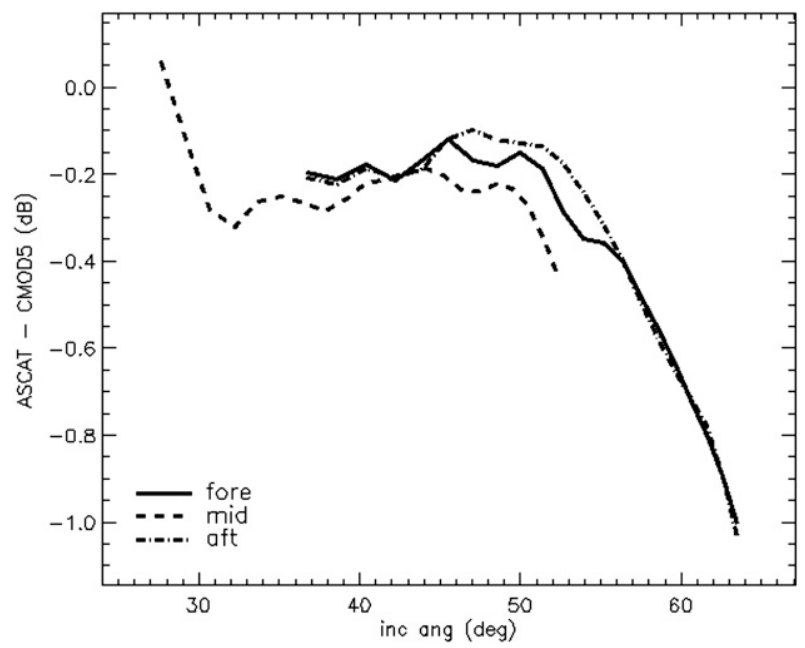

FIG. 14. Mean difference between the backscatter produced by CMOD-5 (with ECMWF analysis winds) and ASCAT data from the right-hand beams over the open ocean in July 2009.

incidence angles rather than an indication of problems in the ASCAT calibration.

Because CMOD-5 forms the basis for many wind vector retrieval algorithms this discrepancy at large incidence angles could potentially lead to large errors in the retrieved wind speed. However, the approach taken by the OSI SAF (Verspeek et al. 2010) circumvents this problem by applying bias correction factors to ASCAT data before wind retrieval.

The ocean validation can also be used to monitor the stability of the ASCAT. Figure 15 shows a time series over several years using the NOC calibration corrections (Verspeek and Stoffelen 2010). Note that the small step change in the calibration of the left midbeam during September 2009 has been provisionally corrected by subtracting $0.125 \mathrm{~dB}$ from September 2009 onward. The ocean calibration residual (difference between measured backscatter and CMOD-5.n-simulated backscatter values obtained from the collocated NWP wind field) is in the order of $0.1 \mathrm{~dB}$. The results from all beams are close together showing that interbeam variations are very small.

A seasonal variation is clearly seen in Fig. 15. This may be due to seasonal changes in the mean wind speed and mean stability at the buoys affecting the mesoscale wind variability. This would then cause some modulation in the spatial representation (wind component) errors as a function of season. As discussed in Stoffelen (1998), the random errors in wind components may cause apparent biases when comparing wind sensing systems with different random error characteristics.

These results show that the ASCAT instrument is very stable over time, although there does appear to be a small downward trend. This may be due to changes in the operational ECMWF model over time (the forecasting system is updated twice a year). To verify such changes, the ASCAT winds are monitored against a set of buoy winds. The buoys cover the whole globe but are located mainly in the Northern Hemisphere and tropics.

Figure 16 shows evidence that over an extended set of Northern Hemisphere and tropical buoy winds collocated with ASCAT, the ECMWF model has been rather stable with a similar seasonal variation each year. There appears to be a small decrease in ASCAT wind speeds over this set of buoys, which is in line with Fig. 15, although further evidence is needed to support such subtle change.

It is also possible to use ocean backscatter to directly monitor the ASCAT calibration without the use of backscatter models, NWP, or buoy winds. Figure 17 shows a section of $0.4-\mathrm{dB}$ width through a three-dimensional plot of the ASCAT backscatter triplets from the open ocean during August 2009. The data points tend to fall into two distinct regions, with higher and lower midbeam backscatter values. The $x$ axis is then divided into bins of $0.4-\mathrm{dB}$ width and the black circles show the location of the peak density of the data in the upper region of each bin. If the position of peak density is calculated for two separate months, then a mean of the differences in the bins can be calculated. Figure 18 shows the mean difference for the months of August and November 2009 as a function of incidence angle, and we find that there has been a change of approximately $0.1 \mathrm{~dB}$ between these two dates.

This approach can also be used to determine the date on which the change took place. If we calculate the position of the peak density using data from August 2009 then the number of ocean triplets in each orbit lying above and below this position should be approximately equal if the calibration remains constant. However, as shown in Fig. 19, a change occurs on 11 September 2009. The cause of this change has not yet been determined but it is not related to an upgrade to the ASCAT level $1 \mathrm{~b}$ processor (which took place several days before this date) or to a satellite maneuver (which took place several days later).

The results presented in this section show that ASCAT data are within $0.2 \mathrm{~dB}$ of the value predicted by CMOD-5.n, with ECMWF-equivalent neutral wind fields over an incidence angle range $25^{\circ}-55^{\circ}$. This is consistent with the expected ASCAT calibration accuracies given by Wilson et al. (2010). Although the differences between the two become larger above $55^{\circ}$ this may not be a reflection of the ASCAT calibration accuracy, but a result of possible inaccuracies of the CMOD-5 model when extrapolated to this incidence angle range. 


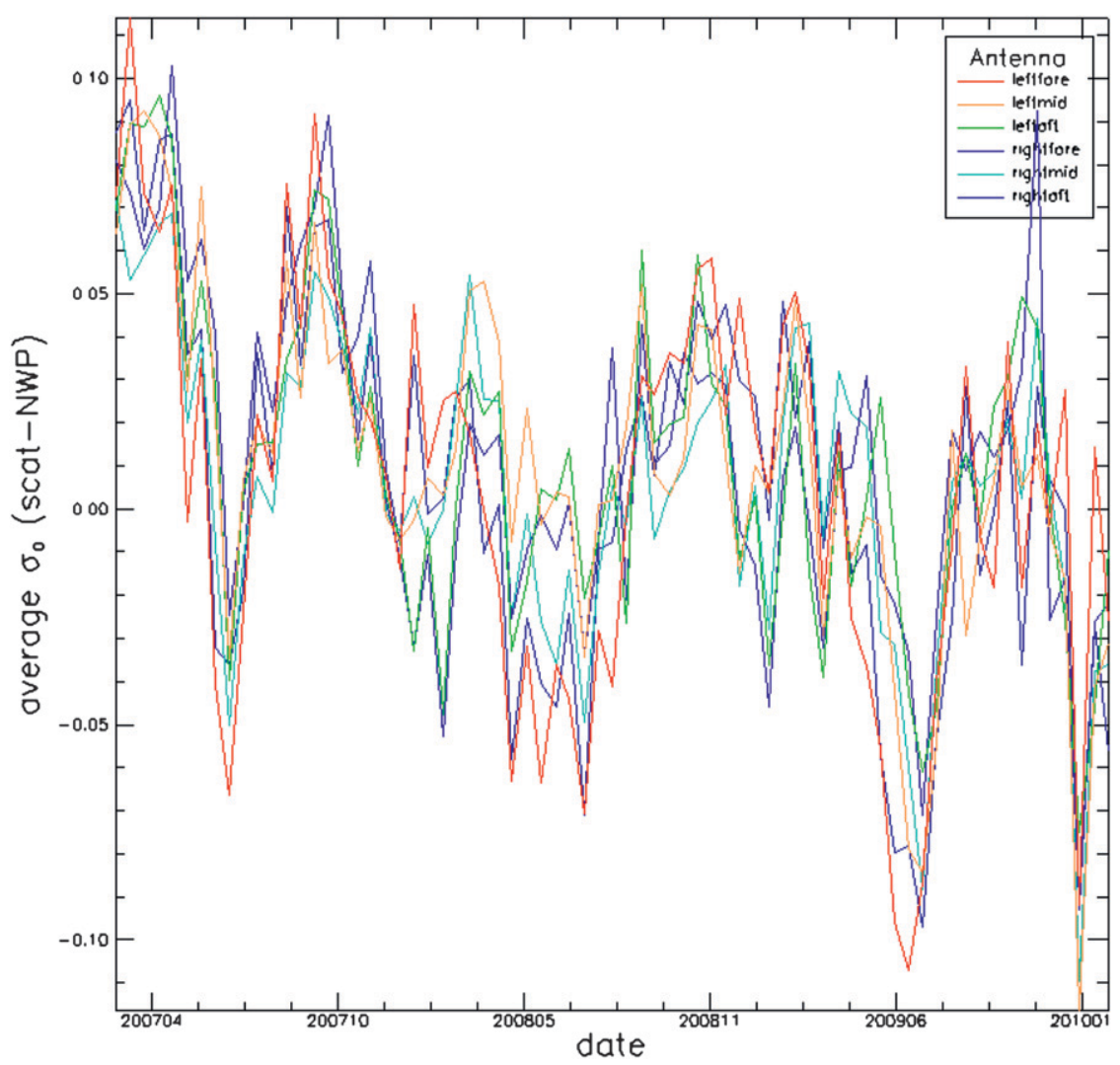

FIG. 15. Time series of ASCAT NWP ocean calibration residuals for each antenna. NOC corrections accumulated from September 2008 through August 2009 are applied (Verspeek and Stoffelen 2010). All level 1B backscatter changes are compensated by reverse corrections (Verspeek et al. 2010).

\section{c. Validation using stable sea ice}

Analysis of data from the ERS scatterometers has shown that backscatter from some regions of sea ice is approximately stable and can be accurately modeled. De Haan and Stoffelen (2001) find that the points given by plotting the fore, mid-, and aft backscatter from stable sea ice in a 3D measurement space form a line, with the position along the line being related to the "age" characteristic of the ice. This ice line model can easily be inverted to retrieve an estimate of the ice age from any backscatter triplet.

Because we do not have prior information about the ice age we cannot use this model to give backscatter values that can be compared to ASCAT data. However, we can compare ASCAT data over stable sea ice to the model to see if they are consistent. Additionally, because sea ice is a relatively stable distributed target, we can use the backscatter from it to investigate the noise characteristics of ASCAT measurements.

To find regions of sea ice we bin ASCAT data in a polar grid and identify the grid cells where the RMS difference between the fore and aft beam backscatter is below a threshold of $0.5 \mathrm{~dB}$. This strategy for locating sea ice is discussed and compared to other methods by Neyt et al. (2004). We then use the ice line model of de Haan and Stoffelen (2001) to retrieve the ice age for all

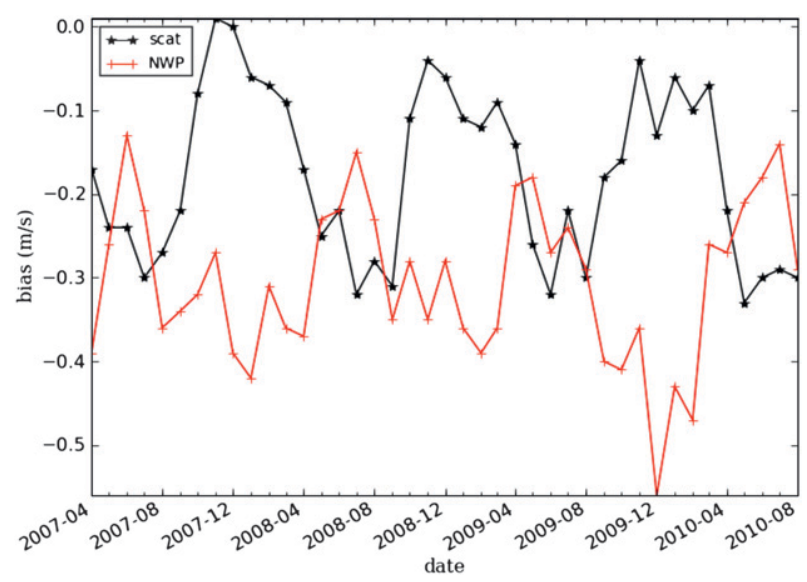

FIG. 16. Time series of ASCAT and NWP buoy wind biases from a triple collocation dataset. Level 2 changes have been compensated and all level 1B backscatter changes are compensated by reverse corrections (Verspeek et al. 2010). 


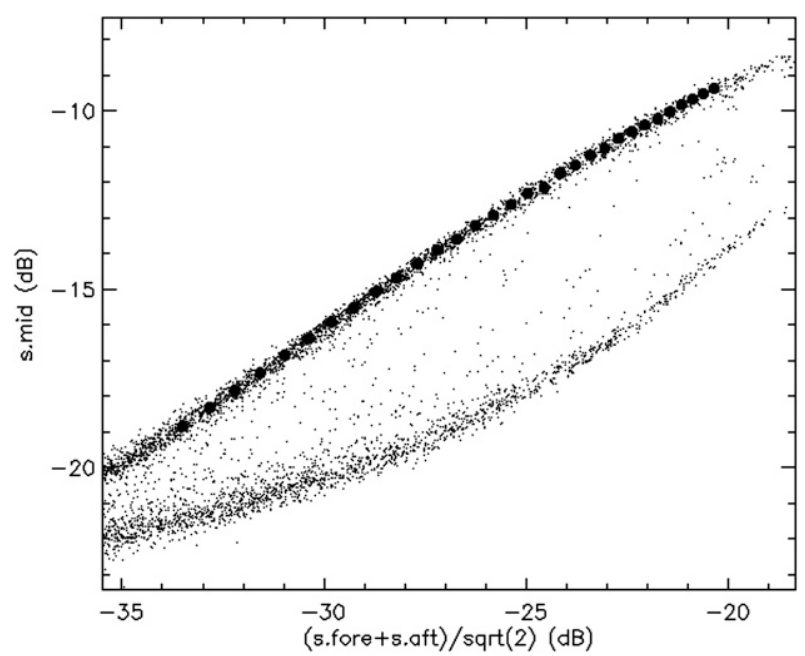

FIG. 17. Section along the $x=y$ axis of a three-dimensional plot where the $x, y$, and $z$ axes correspond to the fore, mid, and aft backscatter from ocean $\sigma_{0}$ triplets. Small points show data from the left-hand beams during August 2009 and large circles show the position of the maximum density of the data points in the upper region in bins along the $x$ axis.

the triplets in cells identified as sea ice. Cells in which the standard deviation of the ice age is below 0.5 are assumed to contain stable sea ice.

Sections through the three-dimensional plot of the resulting stable sea ice triplets are shown in Figs. 20-22 for the near, mid-, and far range of the left-hand swath (i.e., for low, mid-, and high incidence angles).

At low and midrange incidence angles, the ASCAT data lie close to the model line. At larger incidence angles the data and model start to differ. However, because the ice line model was developed from ERS data

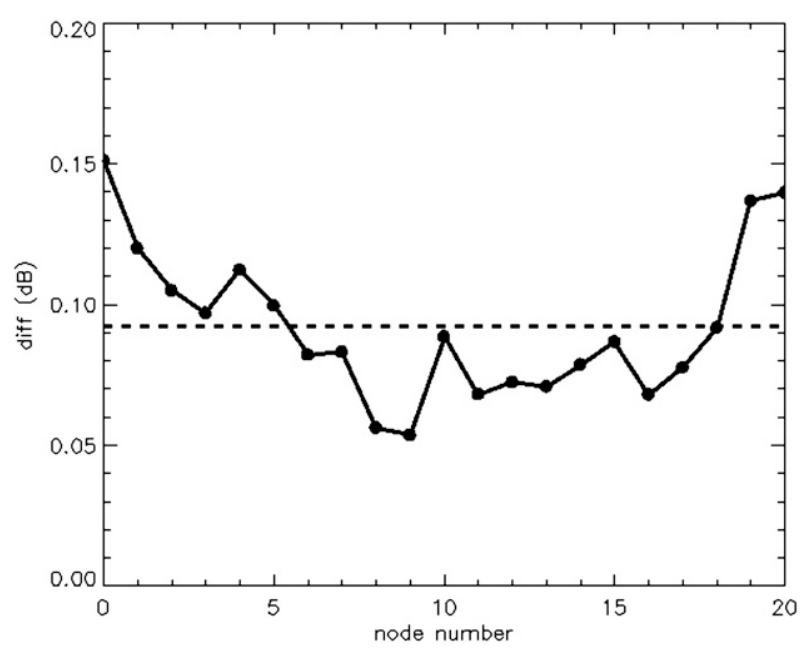

FIG. 18. Mean difference between the positions of maximum density in data from August and November 2009.

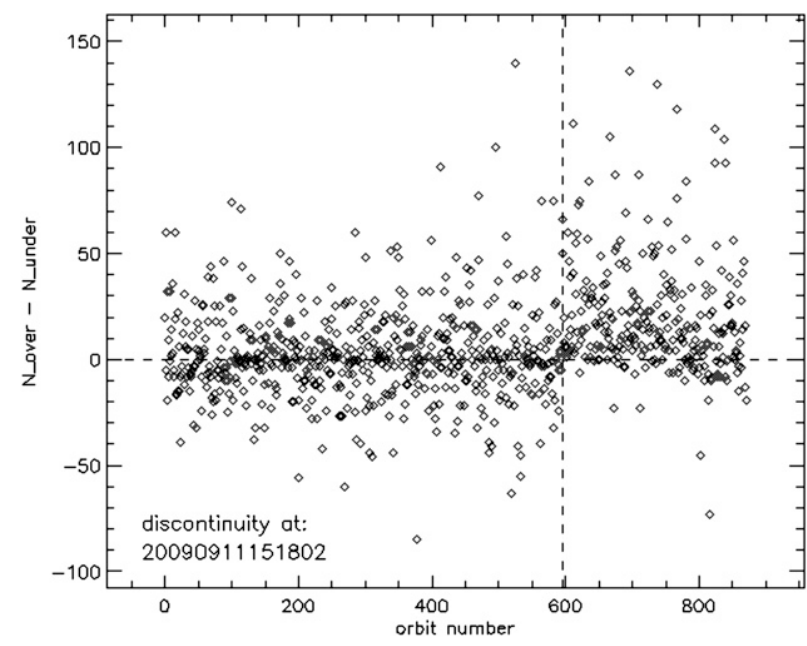

FIG. 19. Difference in the number of ocean triplets above and below the position of maximum density in each orbit during August and September 2009.

covering the incidence angle range $19^{\circ}-55^{\circ}$, discrepancies between model and data above $55^{\circ}$ are likely due to inaccuracies in the extrapolated model.

Fitting a straight line to the backscatter from stable sea ice and calculating the RMS distance between the data and line gives an estimate of noise in ASCAT measurements. Figure 23 shows the noise (converted to normalized standard deviation of the backscatter values $\left.K_{p}\right)$ as a function of incidence angle. This is approximately $4.5 \%$ across the swath, which is close to the expected value of $3 \%-4 \%$.

The results presented in this section show that calibrated ASCAT data from regions of stable sea ice in the Antarctic is consistent with the ice line model at small and medium incidence angles, which gives further

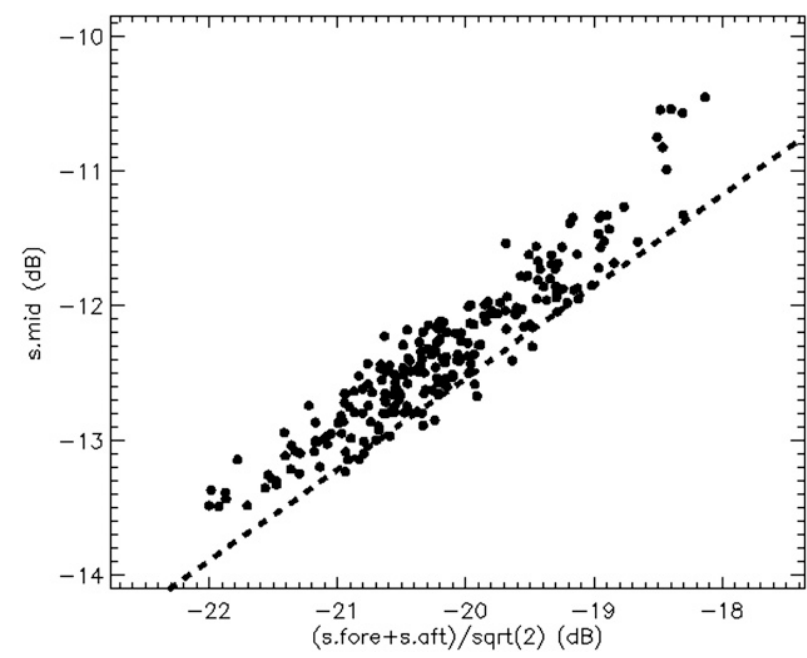

FIG. 20. Backscatter from stable sea ice (circles) compared to the ice line model (dashed line) at the near side of the left-hand swath. 


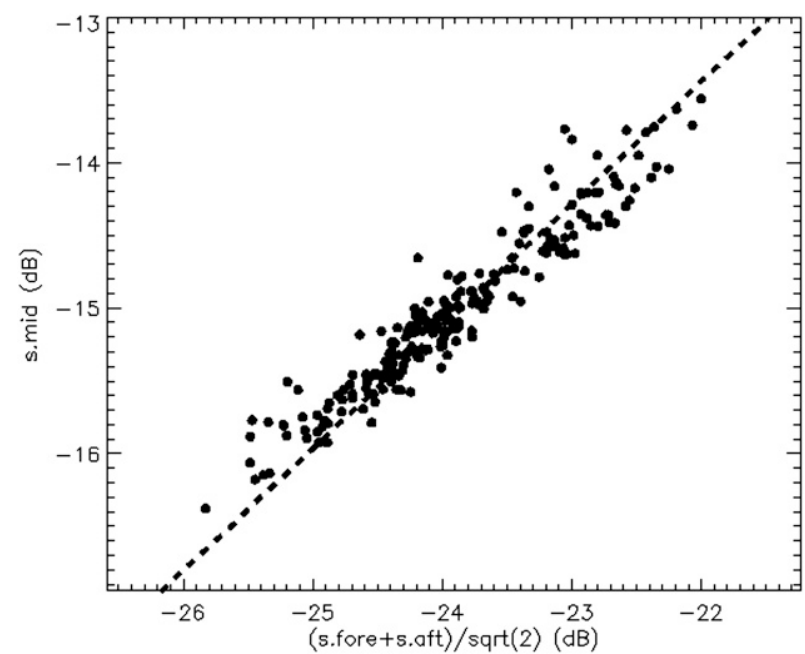

FIG. 21. As in Fig. 20, but at the center of the left-hand swath.

confidence in the accuracy of the ASCAT calibration. At large incidence angles the ASCAT data and the model show discrepancies. However, this does not immediately point to any problem with the ASCAT data because the ice line model has not been validated over $55^{\circ}$.

\section{Overall summary and conclusions}

This paper describes the transponder-based calibration approach for the ASCAT on MetOp-A and presents the results from validations over natural targets using data from the period 2007-09. The expected calibration accuracy of ASCAT has been estimated as $0.15-0.25 \mathrm{~dB}$ (Wilson et al. 2010) through an analysis of the residuals between transponder data and fitted gain patterns.

ASCAT backscatter over the Amazon rainforest has been validated by comparing the isotropic backscatter against the value of $-6.5 \mathrm{~dB}$ given by ERS data. We find that the ASCAT values of $\gamma_{0}$ decreases from -6.2 to $-6.8 \mathrm{~dB}$ over the incidence angle range of $25^{\circ}-65^{\circ}$. This difference in behavior suggests that there may be complications when constructing long-term time series of ERS and ASCAT data. However, the values from all ASCAT beams are within $0.1 \mathrm{~dB}$ of each other, which is consistent with the expected calibration accuracy. Yearly averages of rainforest backscatter are found to be very stable with changes of about $0.02 \mathrm{~dB}$ over the period 2007-09.

ASCAT data over the ocean have been validated by comparing it against the backscatter produced by CMOD-5.n with ECMWF-equivalent neutral wind fields. This shows an approximately constant bias between the two of about $0.2 \mathrm{~dB}$ over incidence angle range $25^{\circ}-55^{\circ}$. This is inconsistent with the rainforest results. Although the data and model difference increases to around $1 \mathrm{~dB}$ at

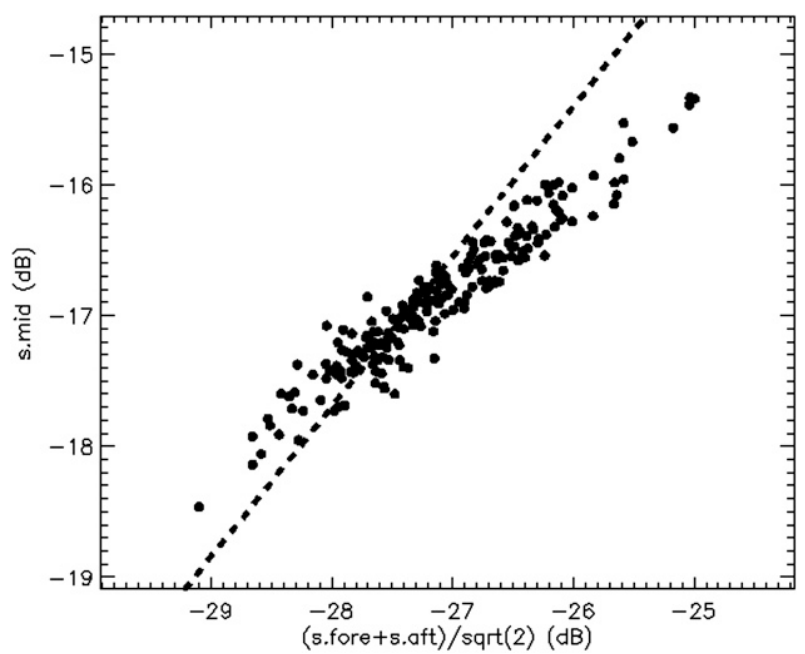

FIG. 22. As in Fig. 20, but at the far side of the left-hand swath.

incidence angles larger than $55^{\circ}$, this is likely due to inaccuracies in CMOD-5.n, which has not been validated at large incidence angles. The relative interbeam calibration is found to be about $0.1 \mathrm{~dB}$.

Data from regions of stable sea ice in the Antarctic has been compared to the ice line model of de Haan and Stoffelen (2001), and the two are found to be consistent except at large incidence angles. However, as with CMOD-5, the ice line model was developed from ERS data and has not been validated over $55^{\circ}$. Hence, the discrepancy is likely due to inaccuracies in the model rather than the ASCAT calibration. An examination of the noise in the backscatter measurements of stable sea ice indicates $K_{p}$ to be approximately $4.5 \%$, which is consistent with the expected value of $3 \%-4 \%$.

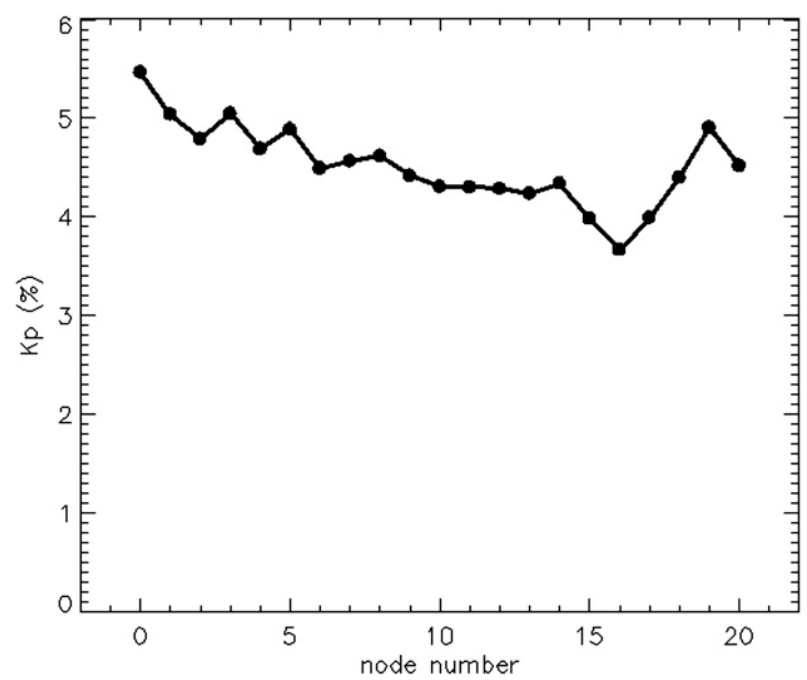

FIG. 23. The $K_{p}$ derived from standard deviation of stable sea ice backscatter around the best fitting straight line. 
The results of these validation techniques are in agreement with the expected calibration accuracy of $0.15-0.25 \mathrm{~dB}$, indicating that the ASCAT calibration has been successful and that ASCAT backscatter products are of high quality. However, there are discrepancies between the various calibration methods: the ocean validation suggests that the difference between ASCAT and ERS data is constant with respect to incidence angle while the rainforest validation suggests an incidence angle dependence. The rainforest validation also points to differences in the behavior of ERS and ASCAT calibrations. These need to be investigated in more detail and understood in order find the optimum method for merging ERS and ASCAT data to create consistent datasets covering long time periods.

Finally, the monitoring of ASCAT using rainforest and ocean data has shown that the instrument is extremely stable. An unexpected but small change in the calibration of the left midbeam occurred in September 2009. The reason for this change is not known and a more detailed analysis of new calibration data is currently underway and will correct any anomalies.

\section{REFERENCES}

Bartalis, Z., 2009: ERS-ASCAT backscatter and soil moisture intercomparison-First results. IPF, Vienna University, ASCAT Soil Moisture Working Note 6.

- W. Wagner, V. Naeimi, S. Hasenauer, K. Scipal, H. Bonekamp, J. Figa, and C. Anderson, 2007: Initial soil moisture retrievals from the METOP-A Advanced Scatterometer (ASCAT). Geophys. Res. Lett., 34, L20401, doi:10.1029/2007GL031088.

de Haan, S., and Stoffelen A., 2001: Ice discrimination using ERS scatterometer. Ocean and Sea Ice SAF Rep. SAF/OSI/KNMI/ TEC/TN/120, 38 pp. [Available online at http://www.knmi.nl/ publications/fulltexts/safosi_w_icescrknmi.pdf.]

EUMETSAT, 2004: ASCAT calibration and validation plan. EUM/EPS/SYS/PLN/01/011, 141 pp.

- 2005: ASCAT level 1 product generation function specification. EUM/EPS/SYS/SPE/990009, 214 pp.

—, 2009a: ASCAT product guide. EUM/OPS-EPS/MAN/04/ 0028, $261 \mathrm{pp}$.

, 2009b: Metop-A ASCAT commissioning quality report. EUM/MET/REP/08/0525, 61 pp.
Figa-Saldaña, J., J. J. W. Wilson, E. Attema, R. Gelsthorpe, M. R. Drinkwater, and A. Stoffelen, 2002: The advanced scatterometer (ASCAT) on the Meteorological Operational (MetOp) platform: A follow-on for European wind scatterometers. Can. J. Remote Sens., 28, 404-412.

Gelsthorpe, R. V., E. Schied, and J. J. W. Wilson, 2000: ASCATMetOp's Advanced Scatterometer. ESA Bull. 102, 9 pp. [Available online at http://www.esa.int/esapub/bulletin/bullet102/ Gelsthorpe102.pdf.]

Hersbach, H., 2003: CMOD5; an improved geophysical model function for ERS $\mathrm{C}$ band scatterometry. ECMWF Tech. Memo. 395, 47 pp. [Available online at http://earth.esa.int/ pub/SCATTEROMETER/ecmwf_rep/cmod5.pdf.]

_ 2008: CMOD5.N: A C-band geophysical model function for equivalent neutral wind. ECMWF Tech. Memo. 554, 22 pp. [Available online at http://earth.eo.esa.int/pcs/ers/scatt/articles/ CMOD5N.pdf.]

Klaes, D., and Coauthors, 2007: An introduction to the EUMETSAT polar system. Bull. Amer. Meteor. Soc., 88, 1085-1096.

Lavergne, T., S. Eastwood, Z. Teffah, H. Schyberg, and L. A. Breivik, 2010: Sea ice motion from low-resolution satellite sensors: An alternative method and its validation in the Arctic. J. Geophys. Res., 115, C10032, doi:10.1029/2009JC005958.

Neyt, X., P. Pettiaux, N. Manise, and M. Acheroy, 2004: Single pass sea-ice discrimination using ERS-2 scatterometer data. Gayana, 68, 432-438.

Portabella, M., and A. Stoffelen, 2009: On scatterometer ocean stress. J. Atmos. Oceanic Technol., 26, 368-382.

Stoffelen, A., 1998: Toward the true near-surface wind speed: Error modeling and calibration using triple collocation. J. Geophys. Res., 103 (C4), 7755-7766.

Verhoef, A., M. Portabella, A. Stoffelen, and H. Hersbach, 2008: CMOD5.n-The CMOD5 GMF for neutral winds. EUMETSAT Ocean and Sea Ice SAF Tech. Note SAF/OSI/CDOP/KNMI/ TEC/TN/165, 13 pp. [Available online at http://www.knmi.nl/ publications/fulltexts/cmod5_neutral_winds_1.0.pdf.]

Verspeek, J., A. Verhoef, and A. Stoffelen, 2010: ASCAT NWP ocean calibration. KNMI Ocean and Sea Ice SAF Rep., 36 pp. [Available online at http://www.knmi.nl/publications/fulltexts/ ascat_nwp_ocean_calibration_1.5_copy1.pdf.]

—, A. Stoffelen, M. Portabella, H. Bonekamp, C. Anderson, and J. Figa-Saldana, 2010: Validation and calibration of ASCAT using CMOD5.n. IEEE Trans. Geosci. Remote Sens., 48, 386-395.

Wilson, J. J. W., and Coauthors, 2010: Radiometric calibration of the advanced wind scatterometer radar ASCAT carried on board the METOP-A satellite. IEEE Trans. Geosci. Remote Sens., 48, 3236-3255.

Zec, J., D. G. Long, and W. Linwood-Jones, 1999: NSCAT normalized radar backscatter coefficient biases using homogeneous land targets. J. Geophys. Res., 104, 11 557-11 568. 